\title{
Loss of constraint on fracture in thin film structures due to creep
}

\author{
R. Huang ${ }^{\mathrm{a}, *}$, J.H. Prévost ${ }^{\mathrm{a}}$, Z. Suo ${ }^{\mathrm{b}}$ \\ ${ }^{a}$ Department of Civil and Environmental Engineering, Princeton University, Princeton, NJ 08544, USA \\ ${ }^{\mathrm{b}}$ Department of Mechanical and Aerospace Engineering, Princeton University, Princeton, NJ 08544, USA
}

Received 18 March 2002; accepted 17 June 2002

\begin{abstract}
Fracture in thin films is normally constrained by the substrates. If the substrate creeps, however, the constraint will be lost over time. This paper presents a two-dimensional model for channel cracks in an elastic film on a viscous layer, and implements an extended finite element method to evolve the displacement field and the stress intensity factor with relatively coarse meshes. Solutions are obtained for stress intensity factors of channel cracks with several in-plane geometries. The stress intensity factor increases with time, indicating the loss of constraint. Several scaling laws are obtained. Extensions of the present model are outlined for nonlinear creeping and viscoelastic layers, as well as a thick viscous substrate. Fracture in thin film structures subjected to ratcheting deformation under cyclic temperatures using the analogy between creeping and ratcheting is also discussed. (C) 2002 Acta Materialia Inc. Published by Elsevier Science Ltd. All rights reserved.
\end{abstract}

Keywords: Thin film; Fracture; Creep

\section{Introduction}

Fracture in a thin film structure is constrained when both the film and the substrate are elastic [13]. Fig. 1 illustrates the influence of constraint on fracture. In Fig. 1(a), a crack of length $a$ has been inserted into a freestanding sheet of thickness $h$, initially under a uniform tensile stress $\sigma$. The displacement in the sheet far away from the crack is held rigidly so that the remote stress does no work

* Corresponding author. Tel.: +1-609-258-1619; fax: +1609-258-1270.

E-mail address: ruihuang@princeton.edu (R. Huang). as the crack grows. After the insertion of the crack, the elastic energy is relaxed in the volume of material around the crack, as indicated by the shaded region in Fig. 1(a). For a freestanding large sheet, the volume is approximately $a^{2} h$, and the change in the elastic energy is $\Delta U_{E}=-$ $\psi_{a}^{2}\left(\sigma^{2} / E\right) a^{2} h$, where $E$ is Young's modulus of the sheet. The dimensionless factor $\psi_{a}$ takes into account the exact geometrical details of the volume of material affected by the crack and can be determined by solving the appropriate elasticity boundary-value problem. The energy release rate $G$ is the amount of the elastic energy decrease associated with the crack advancing per unit area, namely, 
(a)

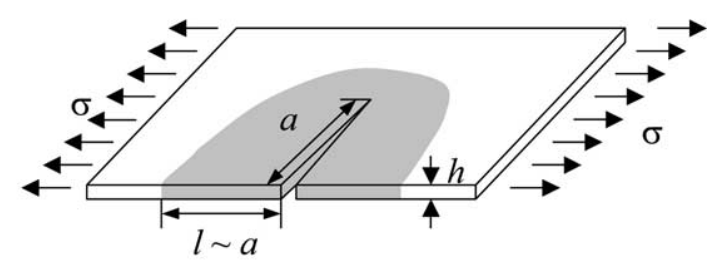

(b)

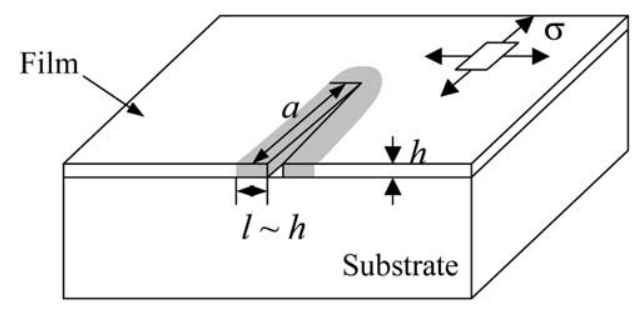

(c)

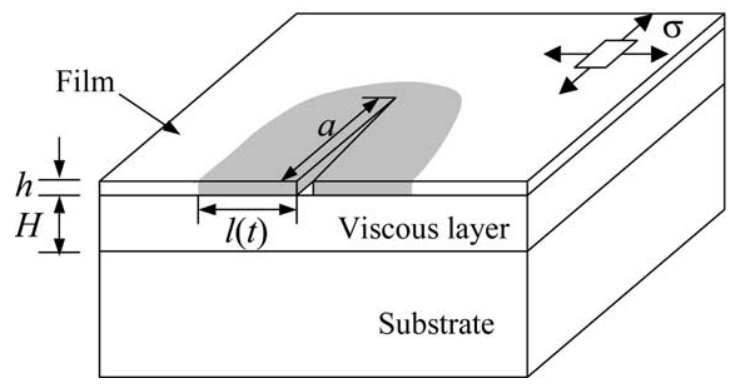

Fig. 1. Schematic diagrams of thin film fracture: (a) A freestanding sheet containing a through-crack under a uniform tensile stress; (b) a thin film bonded to a substrate containing a channel crack; and (c) a thin film on a viscous layer containing a channel crack. In each case, the shaded region indicates the volume of material in which the elastic energy is affected by the crack.

$$
G=2 \psi_{a}^{2} \frac{\sigma^{2}}{E} a
$$

In Fig. 1(b), a channel crack of length $a$ has been inserted into a thin film of thickness $h$ on a substrate. Prior to the insertion of the crack, the film is under a uniform tensile stress $\sigma$. The substrate now constrains the elastic deformation affected by the crack to a length scale of approximately the film thickness. Consequently, the volume in which the elastic energy is relaxed now scales with $a h^{2}$, and the change in the elastic energy becomes $\Delta U_{E}=-\psi_{b}^{2}\left(\sigma^{2} / E\right) a h^{2}$, where the dimensionless factor $\psi_{b}$ characterizes the film-substrate interaction and is independent of the crack length when $a \gg h$. The energy release rate in this case is

$G=\psi_{b}^{2} \frac{\sigma^{2}}{E} h$

A comparison between Eqs. (1) and (2) clearly shows the effect of substrate constraint. For a freestanding film, $G$ scales with the crack length $a$. For a film on an elastic substrate, $G$ scales with the film thickness $h$. In the latter case, as long as the initial crack length exceeds the film thickness, the energy release rate is independent of the crack length. Whether this crack will grow into a long channel crack is independent of the initial flaw size.

Studies have shown that the constraint on fracture is reduced when the substrate deforms plastically $[4,5]$. By the same reasoning, if the substrate creeps, the constraint will be completely lost over time. The loss of constraint becomes an important issue as many organic materials and glasses are used in new devices, e.g. [6,7]. In this paper we consider a thin film structure with a viscous interlayer between the film and the substrate (Fig. 1(c)). The film is elastic and under residual tensile stress $\sigma$. As the interlayer creeps, the volume in which the elastic energy is relaxed by the crack increases and scales with $\operatorname{ahl}(t)$, where $l(t)$ is a length scale increasing with time. The energy release rate $G$ now increases with time, which indicates that the constraint is gradually lost. The plan of this paper is as follows. Section 2 describes a two-dimensional (2D) model for a thin elastic film on a linear viscous layer. Section 3 presents both analytical and numerical solutions for several geometries. Extensions of the present model are discussed in Section 4.

\section{The model}

A 2D model was developed to study the stress relaxation of elastic film islands on viscous substrates [8], and which is outlined below for the 
present study. The model is an extension of the 1D shear lag model $[9,10]$. A similar model has been used to study crack paths in thin elastic films bonded to elastic substrates [11].

Fig. 1(c) shows an elastic film of thickness $h$ on a viscous layer of thickness $H$, which in turn lies on a thick substrate. The thick substrate is assumed to be rigid. The viscous interlayer starts to creep at time $t=0$. Before that, the interlayer is assumed to be rigid, and the film is subjected to a uniform biaxial tensile stress with the associated in-plane strain $\varepsilon_{0}$. A plane stress approximation is used to describe the in-plane deformation of the film, with $u_{\alpha}(x, y, t)$ as the in-plane displacements averaged through the film thickness and measured relative to the uniformly pre-strained state. The total average in-plane strains are

$\varepsilon_{\alpha \beta}=\varepsilon_{0} \delta_{\alpha \beta}+\frac{1}{2}\left(u_{\alpha, \beta}+u_{\beta, \alpha}\right)$.

The greek subscripts, $\alpha$ and $\beta$, take on the values of the in-plane coordinates, $x$ and $y$. Assuming that the material of the thin film is isotropic with Young's modulus $E$ and Poisson's ratio $v$, the average in-plane stresses are

$\sigma_{\alpha \beta}=\frac{E}{1-v^{2}}\left[(1-v) \varepsilon_{\alpha \beta}+v \varepsilon_{\gamma} \delta_{\alpha \beta}\right]$.

We adopt the convention that a repeated greek subscript implies summation over the two inplane dimensions.

In addition to the in-plane stresses, the film is subjected to shear tractions $\tau_{\alpha}$ along the interface between the film and the viscous layer. For a thin viscous layer, we assume that the flow velocities are linearly distributed across the layer thickness. At the film-interlayer interface, the velocities equal to the in-plane velocities of the film. At the interlayer-substrate interface, the velocities are zero. For the time being, we assume that the interlayer is linearly viscous with viscosity $\eta$. The shear tractions relate to the strain-rates as

$\tau_{\alpha}=\frac{\eta \partial u_{\alpha}}{H \partial t}$.

Force balance of a differential element of the elastic film requires that $\sigma_{\alpha \beta, \beta}-\tau_{\alpha} / h=0$.

Eq. (6) can also be obtained by integrating the 3D force balance equations over the thickness of the film and interpreting $\sigma_{\alpha \beta}$ as the average stress across the film thickness. Note that Eq. (6) is equivalent to the $2 \mathrm{D}$ equilibrium equation for a plane stress problem with a body force of magnitude $-\tau_{\alpha} / h$.

In terms of the displacements, Eq. (6) becomes

$\frac{\partial u_{x}}{\partial t}=D\left[\frac{\partial^{2} u_{x}}{\partial x^{2}}+\frac{1-v \partial^{2} u_{x}}{2 \partial y^{2}}+\frac{1+v \partial^{2} u_{y}}{2 \partial x \partial y}\right]$,

$\frac{\partial u_{y}}{\partial t}=D\left[\frac{\partial^{2} u_{y}}{\partial y^{2}}+\frac{1-v \partial^{2} u_{y}}{2 \partial x^{2}}+\frac{1+v \partial^{2} u_{x}}{2 \partial x \partial y}\right]$,

where

$D=\frac{E h H}{\left(1-v^{2}\right) \eta}$.

Eqs. (7) and (8) evolve the displacement field, analogous to the diffusion equations, with $D$ as the effective diffusivity.

Now consider a straight channel crack in the elastic film, aligned parallel to the $x$-axis. The mode I and mode II stress intensity factors at the crack tip are related to the energy release rate by the classical plane stress relation $G=\left(K_{\mathrm{I}}^{2}+\right.$ $\left.K_{\mathrm{II}}^{2}\right) / E$. Without any body force, the energy release rate coincides with a path-independent $J$-integral:

$J=\int_{C}\left(W n_{1}-\sigma_{\alpha \beta} n_{\beta} u_{\alpha, 1}\right) \mathrm{d} s$,

where $C$ is any contour circling the crack tip in the counter-clockwise direction with $n_{\alpha}$ as its outward unit normal and $\mathrm{d} s$ as its arc length, and $W$ is the strain energy per unit area per unit thickness of the film, i.e. $W=\sigma_{\alpha \beta} \varepsilon_{\alpha \beta} / 2$. With a body force $-\tau_{\alpha} / h$, as in Eq. (6), however, the conventional $J$-integral is no longer path-independent. We introduce and define a new integral

$J_{B}=\int_{C}\left(W n_{1}-\sigma_{\alpha \beta} n_{\beta} u_{\alpha, 1}\right) \mathrm{d} s+\frac{1}{h} \int_{A} \tau_{a} u_{a, 1} \mathrm{~d} A$,

where $A$ is the area bounded by the contour $C$ and the crack. It can be shown that the new integral $J_{B}$ 
is indeed path-independent. Near the crack tip, the stress filed is singular in $x$ and $y$, but smooth in $t$. Consequently, in Eqs. (7) and (8), the terms on the right-hand side are more singular than those on the left-hand side, and the form of the singular field is determined by setting the right-hand side to zero. The latter is identical to the governing equations for the 2D elastic field without any body force. Thus, the presence of the body force does not change the character of the dominant singular behavior at the crack tip, and the gradient of the displacements has the conventional inverse square root singularity at the tip (i.e. $u_{\alpha, 1} \sim r^{-1 / 2}$ ), so that the area integral at the right-hand side of Eq. (11) is nonsingular. As the contour $C$ approaches the crack tip, the area integral approaches zero and $J_{B}$ approaches $J$. The new integral $J_{B}$ coincides with the energy release rate $G$.

In the remainder of this paper, the above 2D model is solved by using the finite element method. The finite element software DYNAFLow[12] is used. First, the evolving displacement field is solved from Eqs. (7) and (8). At each time step, the strain and stress are computed from Eqs. (3) and (4), respectively. The presence of a crack is modeled by using the extended finite element method [13-16], in which discontinuous enrichment functions are added to the conventional finite element framework to account for the discontinuity across the crack. Given the displacement, strain, and stress fields at certain time, the stress intensity factors are computed using the domain form of the interaction integrals [13]. For the present problem, the interaction integrals are derived from the new integral, $J_{B}$, instead of the conventional $J$-integral used in Ref. [13]. All the results presented subsequently are for mode I fracture, but the approach can readily be extended for general mixed mode fracture. While other numerical methods may be applied to solve the problems and to compute the stress intensity factor as well, the advantage of the present approach using the extended finite element method is that it allows the crack to be arbitrarily aligned within the mesh and produces excellent accuracy with a relatively coarse mesh.

\section{Analytical and numerical solutions}

This section considers three crack geometries (Fig. 2). In all cases, the edges of the films are traction-free. In addition to the numerical results, several analytical scaling laws for the stress intensity factor are deduced.

\subsection{A semi-infinite straight crack in an infinite film}

Consider an infinite film with an isolated semiinfinite straight crack along the negative $x$-axis with the crack tip at $x=0$. At $t=0$, the stress field in the film is uniform, the displacement is zero everywhere, and the stress intensity factor at the crack tip is zero. As the viscous layer creeps, the stress field in the film relaxes in the crack wake, but intensifies around the crack tip. The stress intensity factor $K_{\mathrm{I}}$ linearly depends on the biaxial stress, $\sigma_{0}=E \varepsilon_{0} /(1-v)$. The problem lacks any fixed length scale. (The thicknesses, $h$ and $H$, enter the model through the quantity $D$ in Eq. (9) and do not set the length scale of the problem.) Instead, there exists a time-dependent length scale associated with creep, $(D t)^{1 / 2}$. Consequently, by dimensional analysis, the stress intensity factor takes the form

$K_{\mathrm{I}}(t)=\kappa E \varepsilon_{0}(D t)^{1 / 4}$,

where $\kappa$ is a dimensionless number depending only on Poisson's ratio $v$.

Numerical solutions are obtained for a crack of length $a$ in a film of size $2 a \times 2 a$, as shown in Fig. 2(a). The evolution of the displacement field is similar to that in relaxation of a strained elastic film on a viscous layer [8], starting from the traction-free surfaces (including the crack faces) and diffusing into the film. The relaxation along the crack faces leads to opening of the crack with intensified stress around the crack tip. The crack length $a$ is chosen such that $a \gg\left(D t_{\mathrm{m}}\right)^{1 / 2}$, where $t_{\mathrm{m}}$ is an arbitrarily selected final time for computation. For the time $t<t_{\mathrm{m}}$, the crack tip field is not affected by the relaxation from the outer boundaries, simulating a semi-infinite crack in an infinite film. Fig. 3 shows the normalized stress intensity factor, $\bar{K}_{\mathrm{I}}=K_{\mathrm{I}} / E \varepsilon_{0}\left(D t_{\mathrm{m}}\right)^{1 / 4}$, as a function of the nor- 
(a)

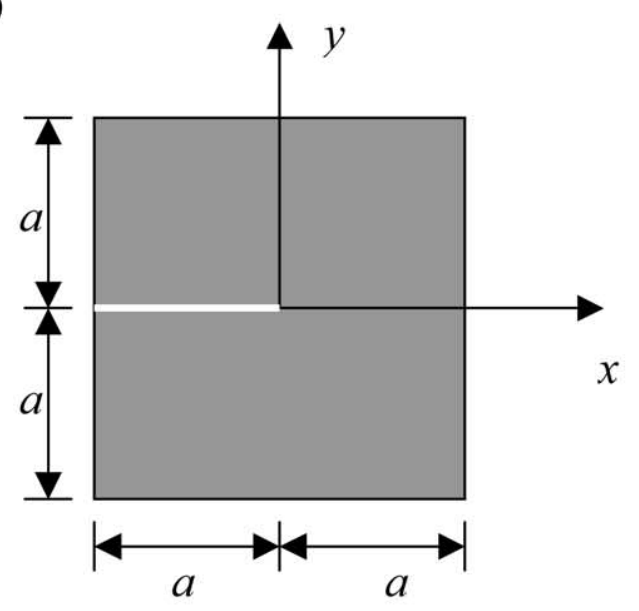

(b)

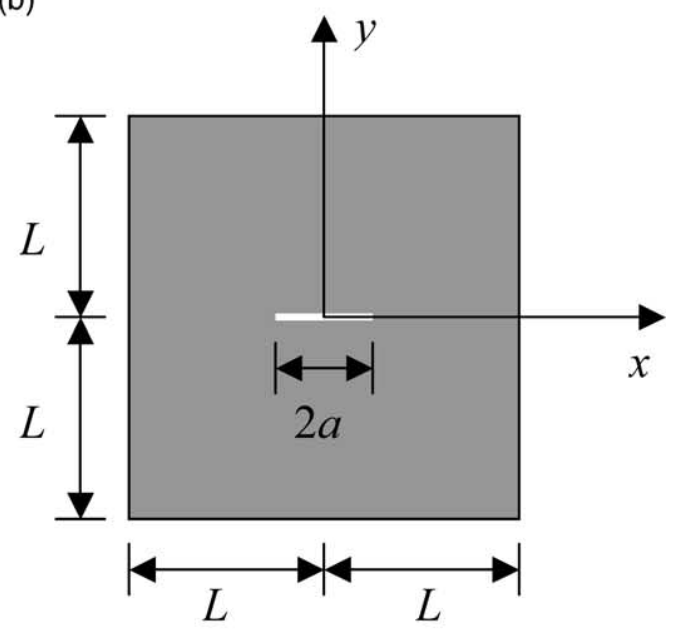

(c)

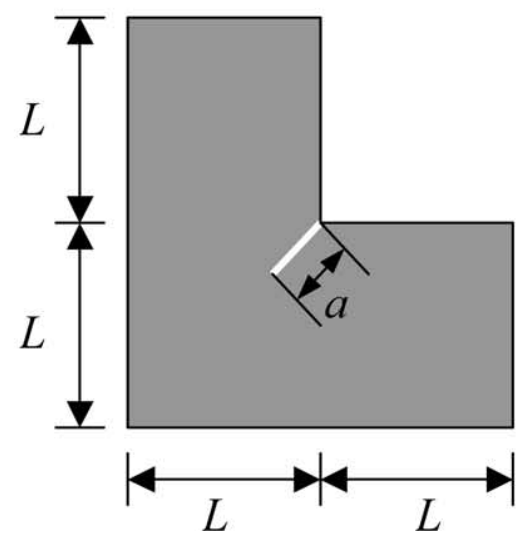

Fig. 2. (a) A edge crack of length $a$ in a square film of size $2 a \times 2 a$, which simulates a semi-infinite crack as $a \rightarrow \infty$; (b) a center crack of length $2 a$ in a square film of size $2 L \times 2 L$, which simulates a finite crack in an infinite film as $L / a \rightarrow \infty$; and (c) a corner crack of length $a$ in an L-shaped film.

malized time, $\bar{t}=t / t_{\mathrm{m}}$, plotted in the logarithmic scales for various Poisson's ratios. The results are independent of $a$ when $a>5\left(D t_{\mathrm{m}}\right)^{1 / 2}$ and can be considered as for a semi-infinite crack in an infinite film. As predicted by Eq. (12), the stress intensity factor increases with time, fitting into parallel straight lines in the logarithmic plot with the slope equal to 1/4 for all Poisson's ratios. The dimensionless number $\kappa$ equals to the normalized stress intensity factor at $\bar{t}=1$, and is listed in Table 1 .

For a semi-infinite crack in an infinite film bonded to an elastic substrate, the stress intensity factor takes the form $[2,11]$

$K_{\mathrm{I}}^{E}=\psi E \varepsilon_{0}(h)^{1 / 2}$

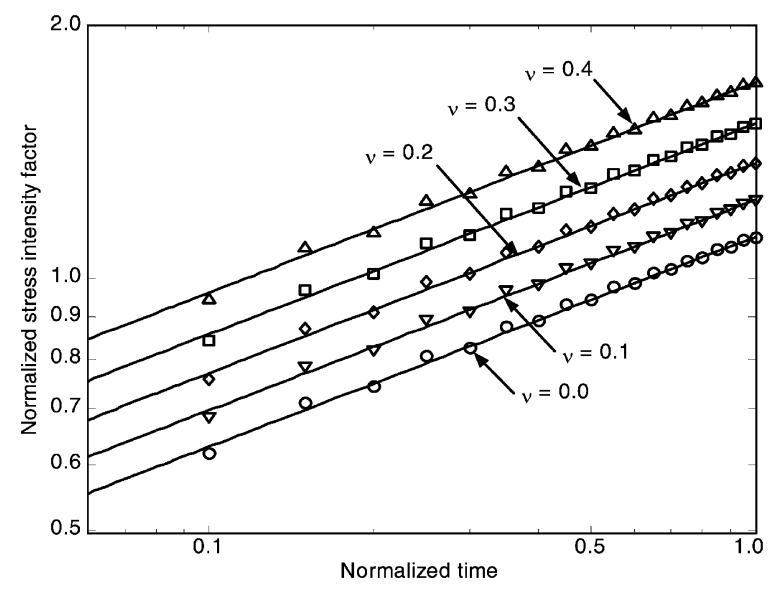

Fig. 3. Normalized stress intensity factor $\bar{K}_{I}=$ $K_{I} / E \varepsilon_{0}\left(D t_{\mathrm{m}}\right)^{1 / 4}$ as a function of the normalized time $\bar{t}=t / t_{\mathrm{m}}$ for a semi-infinite crack in an infinite film bonded to a viscous layer. The straight lines are predicted by Eq. (12), and the open shapes are the numerical results.

Table 1

Dimensionless number $\kappa$ in Eq. (12) for different Poisson's ratios

\begin{tabular}{llllll}
\hline$v$ & 0.0 & 0.1 & 0.2 & 0.3 & 0.4 \\
$\kappa$ & 1.121 & 1.239 & 1.372 & 1.527 & 1.711 \\
\hline
\end{tabular}


where $\psi$ is a dimensionless number, depending on the elastic mismatch between the film and the substrate. The value of $\psi$, numerically computed in Ref. [2], ranges from less than 1 for a very compliant film on a relatively stiff substrate to more than 5 for a stiff film on a relatively compliant substrate. Nevertheless, the stress intensity factor scales with the square root of the film thickness, and the fracture is constrained by the elastic substrate. However, with a creeping interlayer between the film and the substrate, the present study (Eq. (12)) shows that the stress intensity factor monotonically increases with time, and the value can be much larger than the constrained stress intensity factor $K_{\mathrm{I}}^{E}$ (Eq. (13)) after certain time. That is, due to creep, the constraint on fracture in the thin film is gradually lost over time.

\subsection{A finite crack in an infinite film}

Next, we consider a finite crack of length $2 a$ in an infinite film. In this case, analytical solutions for the stress intensity factor are available at two limiting points. At the very beginning, when $(D t)^{1 / 2} \ll a$, the stress intensity factor follows Eq. (12) for a semi-infinite crack. At the other limit, when $(D t)^{1 / 2} \gg a$, the film reaches equilibrium under the remote biaxial stress, and the stress intensity factor approaches the equilibrium value for a freestanding film under the same remote stress, namely

$K_{\mathrm{I}}^{\infty}=\frac{E \varepsilon_{0}}{1-v}(\pi a)^{1 / 2}$.

Therefore, the stress intensity factor first increases with time, following Eq. (12), and then asymptotically approaches the equilibrium value in Eq. (14).

Numerical solutions are obtained for a square film of size $2 L \times 2 L$ with various $L$, as shown in Fig. 2(b). The solutions are expected to approach the solution for an infinite film as $L / a \rightarrow \infty$. For a finite $L / a$, however, stress relaxation from the edges will eventually reach the crack. As a result, the crack will gradually close down and the stress intensity factor at the crack tips will decrease. After a sufficiently long time, the film is fully relaxed, the crack is completely closed, and the stress intensity factor becomes zero. Fig. 4 shows the numerical results for $L / a=5$. The normalized stress intensity factor versus the normalized time is plotted. Four points are labeled along the curve as $\mathrm{A}, \mathrm{B}, \mathrm{C}$, and $\mathrm{D}$, for which the corresponding distributions of the normalized mean stress, $\left(\sigma_{x x}+\sigma_{y y}\right) /\left(2 \sigma_{0}\right)$, are plotted in the linear gray scale contour. At $t=0$, the film is under uniform biaxial tensile stress and the stress intensity factor at the crack tip is zero. As the viscous layer starts to creep, the biaxial tensile stress drives the crack to open. At point A, stress relaxation starts along the crack wake and the film edges while most part

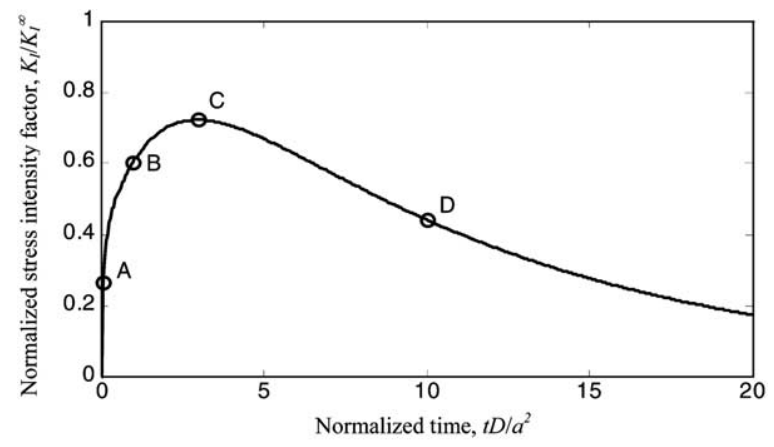

(A)

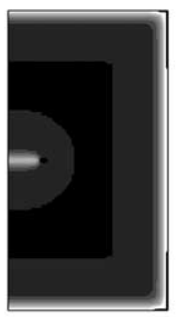

(C)

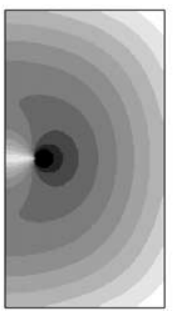

(B)

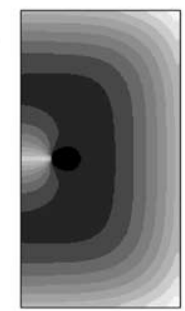

(D)

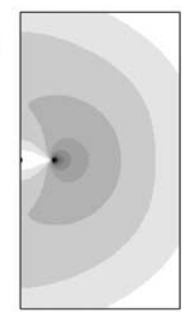

Fig. 4. Numerical results for a square film with a center crack: normalized stress intensity factor as a function of the normalized time, and the distributions of the normalized mean stresses corresponding to the points A, B, C, and D. The amplitude of the normalized stress is visualized in the linear gray scale color map between white (0) and black (1). 
of the film in between remains biaxially stressed; at the crack tip, the stress intensity factor starts to increase. At point $\mathrm{B}$, stress relaxation has reached most part of the film, but there is still part of the film that is not affected and remains the initial state; The stress intensity factor keeps increasing with time. At point $\mathrm{C}$, stress relaxation has reached the whole film, and the stress intensity factor starts to decrease. At point $\mathrm{D}$, the stress magnitude has dropped significantly in most parts of the film due to stress relaxation; the stress intensity factor has dropped to about half of that at point $\mathrm{C}$ and keeps decreasing with time. Eventually the film will be fully relaxed and the stress intensity factor will become zero.

Although the film size in the above numerical solutions is relatively small, the trend is similar for any film sizes. Fig. 5 shows the stress intensity factor as a function of the time for various film sizes, plotted in logarithmic scales. Also plotted in Fig. 5 is the solution for a semi-infinite crack given by Eq. (12), as shown by the straight dashed line with the slope equal to $1 / 4$. The corresponding value of $\kappa$ for $v=0.3$ from Table 1 is used. The horizontal dashed line corresponds to the equilibrium value for an infinite freestanding film. For all film sizes, the stress intensity factor first increases with time, and then decreases. A larger film takes longer time for stress relaxation to affect the stress intensity factor and thus reaches a larger maximum stress intensity factor. The equilibrium value of the stress

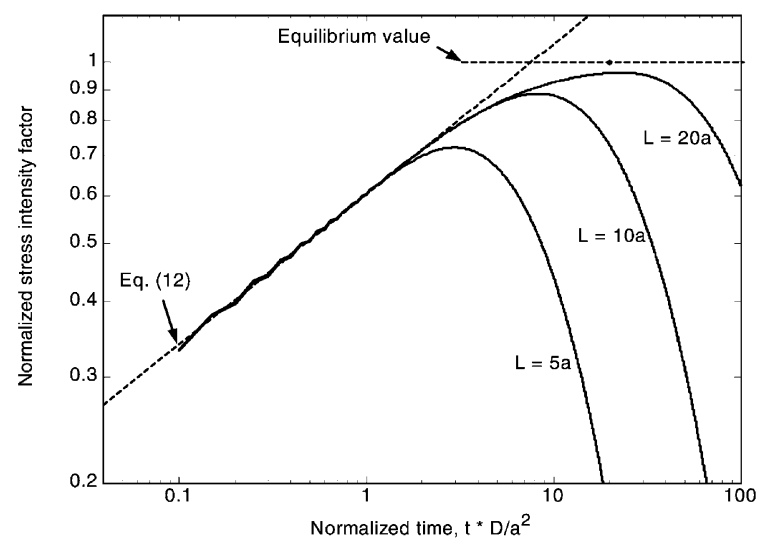

Fig. 5. Normalized stress intensity factor $\bar{K}_{\mathrm{I}}=(1-$ v) $K_{\mathrm{I}} / E \varepsilon_{0}(\pi a)^{1 / 2}$ as a function of the normalized time $\bar{t}=$ $D t / a^{2}$ for a finite center crack in a square film of different sizes. intensity factor given by Eq. (14) presents an upper bound for the maximum stress intensity factor. For a very large film, the stress intensity factor approaches the equilibrium value asymptotically before it decreases due to the effect of stress relaxation.

Comparing to a thin film bonded to an elastic layer, with the presence of a viscous interlayer, the stress intensity factor of a crack in the film increases with time and may reach a much larger value due to the loss of constraint. On the other hand, the creeping of the interlayer relaxes the stress in the film, which eventually reduces the stress intensity factor. One implication of such behaviors is that the crack may grow when the stress intensity factor reaches a critical value, and then arrest in the film as the stress intensity factor drops below the critical value.

\subsection{A crack in an L-shaped film}

Fig. 2(c) shows an L-shaped film with a crack at the corner. For a freestanding L-shaped film, without introducing any crack, the film behaves like a homogenous material with a $90^{\circ}$ wedge cut. Under a symmetric load, the stress is singular near the corner, scaling with the distance $r$ from the corner as $\sigma=k r^{-\lambda}$ [17]. The singularity exponent $\lambda$ is the solution to a transcendental equation,

$\sin [(1-\lambda)(2 \pi-\theta)]-(1-\lambda) \sin \theta=0$,

where $\theta$ is the wedge angle and $0<\lambda \leq 0.5$. For a straight crack, the angle is zero and $\lambda=0.5$. For a $90^{\circ}$ wedge angle, $\lambda=0.456$. The coefficient $k$ depends on the geometry and the remote load. From dimensional analysis, we have $k \propto \sigma_{\infty} l^{\lambda}$, where $\sigma_{\infty}$ is the remote stress and $l$ is a characteristic length from geometry. For a freestanding L-shaped film in Fig. 2(c), we take $l=L$. Now, if we introduce a crack of length $a(a \ll L)$ at the corner, as shown in Fig. 2(c), the stress intensity factor at the crack tip should be proportional to $k$ and takes the form

$K_{I}=\zeta \sigma_{\infty}(a)^{1 / 2}\left(\frac{L}{a}\right)^{\lambda}$

where $\zeta$ is a dimensionless number to be determined from more detailed analysis. 
If the L-shaped film is bonded to an elastic substrate, the crack is constrained and the stress intensity factor will be reduced. When the crack length $a$ is much bigger than the film thickness $h$, the crack reaches the steady state, and the stress intensity factor is proportional to $(h)^{1 / 2}$ but independent of $a$ and the film size $L$. The stress intensity factor of the constrained crack is much below the unconstrained value in Eq. (16). However, the constraint will be lost with a creeping interlayer between the film and the substrate. At the beginning, when $(D t)^{1 / 2} \ll a$, the crack-tip field is not affected by the film geometry and the crack can be considered semi-infinite such that the stress intensity factor essentially follows Eq. (12). Later, when $(D t)^{1 / 2} \gg a$, there are two possibilities: (1) If $(D t)^{1 / 2} \ll L$, the film size $L$ is irrelevant as far as the corner crack is concerned, and the characteristic length is given by $l=(D t)^{1 / 2}$. Following the same argument for the freestanding film, the stress intensity factor at the crack tip takes the form

$K_{\mathrm{I}}(t)=\zeta E \varepsilon_{0}(a)^{1 / 2}\left(\frac{D t}{a^{2}}\right)^{\lambda / 2}$.

(2) If $(D t)^{1 / 2} \geq L$, stress relaxation causes the stress intensity factor to decrease. Eventually, the film is fully relaxed and the stress intensity factor becomes zero. Therefore, with a creeping interlayer, the stress intensity factor first increases and then decreases with a maximum value depending on the film size $L$.

Fig. 6 shows the numerical solutions for the stress intensity factor as a function of time for various ratios between $L$ and $a$. The same normalization as in Fig. 5 is used for the stress intensity factor and the time. Eq. (12) is also plotted in Fig. 6 as the dashed line, using the corresponding $\kappa$ values from Table 1. For all film sizes, the stress intensity factor follows Eq. (12) at the initial stage. When $L \gg a$, we expect to see that the stress intensity factor follows Eq. (17) when $a \ll(D t)^{1 / 2} \ll L$. However, this is not clear from our calculations, even for $L=100 a$ (not shown in Fig. 6). One possible reason is that, from Eq. (12) to Eq. (17), the slope changes from 0.25 to 0.228 , which may be too small to be seen in Fig. 6 .

While Fig. 6 looks similar to Fig. 5, there is one difference. The maximum stress intensity factor in

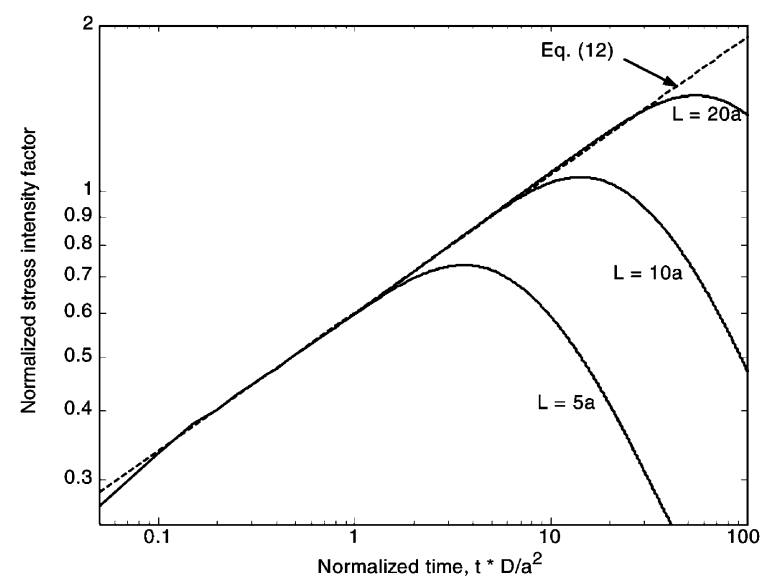

Fig. 6. Normalized stress intensity factor $\bar{K}_{\mathrm{I}}=(1-$ v) $K_{\mathrm{I}} / E \varepsilon_{0}(\pi a)^{1 / 2}$ as a function of the normalized time $\bar{t}=$ $\mathrm{D} t / \mathrm{a}^{2}$ for a corner crack in an L-shaped film of different sizes.

Fig. 5 has an upper bound given by the equilibrium value in Eq. (14), but there is no such bound in Fig. 6 simply due to the singularity of the L-shape. Thus, the stress intensity factor can reach a much larger value and the crack is more likely to grow at the corner of an L-shaped film.

\section{Discussions and extensions}

In this section, we discuss possible extensions of the present model. Based on the analysis in Section 3, we present the corresponding analytical solutions at limiting cases, but leave the full numerical solutions for subsequent studies.

\subsection{Nonlinear creeping model}

The present model assumes a linear viscous creeping interlayer between the film and the substrate. One may extend the model to have a nonlinear creeping interlayer. For example, by using the power law creeping model, Eq. (5) becomes

$\tau_{\alpha}=\left(\frac{\beta \partial u_{\alpha}}{H \partial t}\right)^{1 / n}$,

where $\beta$ has the unit of (stress) $)^{n} \times$ time and $n$ is a dimensionless number. Replacing Eq. (5) with Eq. (18), the model yields a time-dependent length: 
$l=\left[\frac{E^{n} h^{n} H t}{\left(1-v^{2}\right)^{n} \beta}\right]^{1 /(n+1)}$.

Thus, for a semi-infinite crack, Eq. (12) becomes

$K_{\mathrm{I}}(t)=\kappa E \varepsilon_{0}\left[\frac{E^{n} h^{n} H t}{\left(1-v^{2}\right)^{n} \beta}\right]^{1 / 2(n+1)}$.

Similarly, for a corner crack in an L-shaped film, Eq. (17) becomes

$K_{\mathrm{I}}(t)=\zeta E \varepsilon_{0}(a)^{1 / 2}\left[\frac{E^{n} h^{n} H t}{\left(1-v^{2}\right)^{n} \beta a^{n+1}}\right]^{\frac{\lambda}{(n+1)}}$.

When $n=1$ and $\beta=\eta$, Eqs. (20) and (21) reduce to Eqs. (12) and (17), respectively.

\subsection{Linear viscoelastic models}

Another alternative of the material property of the interlayer is linear viscoelastic. We consider two simple models of viscoelasticity. First, using the Kelvin/Voigt viscoelastic model, Eq. (5) becomes

$\tau_{\alpha}=\frac{\eta \partial u_{\alpha}}{H \partial t}+\frac{\mu}{H} u_{\alpha}$

where $\mu$ denotes the elastic shear modulus of the interlayer. Comparing the two terms on the righthand side of Eq. (22), we have a time scale:

$t *=\eta / \mu$.

When $t \ll t *$, the viscous term (i.e. the first term) dominates. For a semi-infinite crack, the stress intensity factor follows Eq. (12) in this regime. When $t \gg t *$, the elastic term (i.e. the second term) dominates, and the model reduces to that for a thin film bonded to an elastic substrate [11]. Therefore, the stress intensity factor will saturate at an equilibrium value determined by the elasticity. For a semi-infinite crack, from dimensional analysis, the equilibrium value takes the form

$K_{\mathrm{I}}^{\mathrm{e}} \propto E \varepsilon_{0}\left(\frac{E h H}{\mu}\right)^{1 / 4}$.

Fig. 7(a) shows the schematic of the stress intensity factor as a function of time for a semi-infinite crack if the interlayer is Kelvin/Voigt-type viscoelastic.

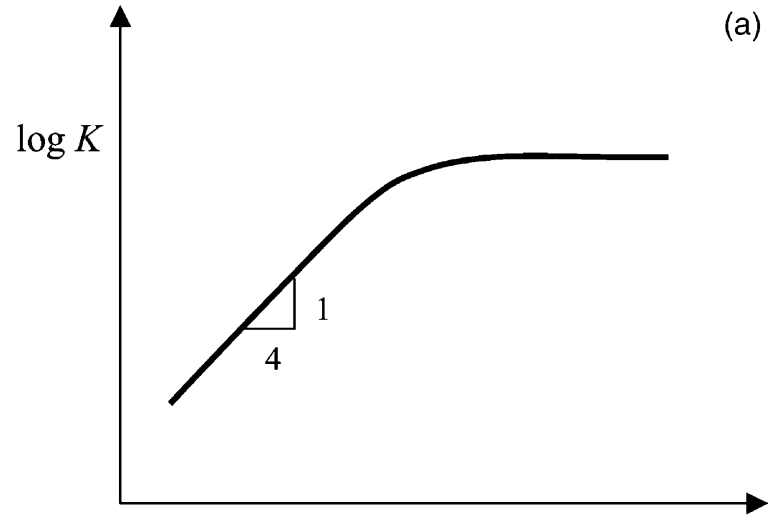

$\log t$

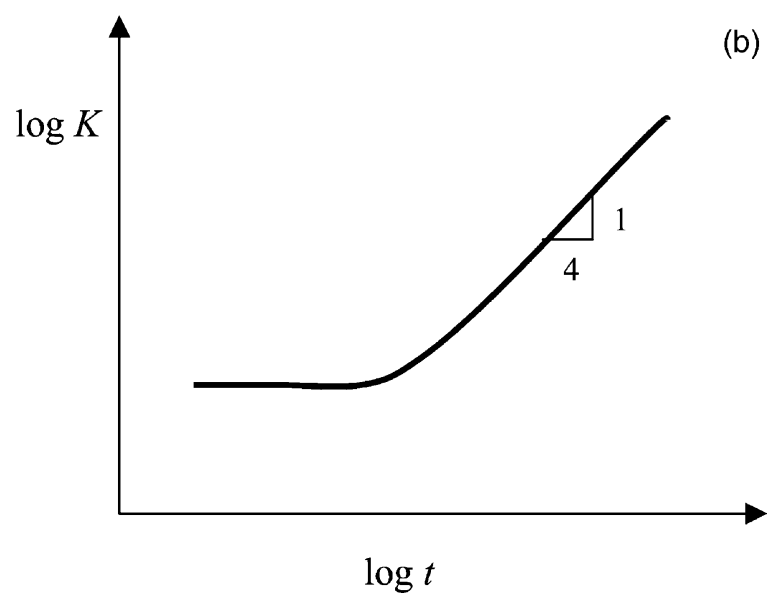

Fig. 7. Schematic $K$-histories of viscoelastic models: (a) Kelvin/Voigt model and (b) Maxwell model.

Alternatively, if we use the Maxwell model of viscoelasticity, Eq. (5) becomes

$\tau_{\alpha}=\mu\left(\frac{u_{\alpha}}{H}-\gamma_{\alpha}^{p}\right)$

where $\gamma_{\alpha}^{p}$ is the viscous portion of the total shear strain, and

$\frac{\partial \gamma_{\alpha}^{p}}{\partial t}=\frac{\tau_{\alpha}}{\eta}$.

Combining Eq. (25) with the film equilibrium equation (Eq. (6)), one can solve for the shear traction $\tau_{\alpha}$ with $\gamma_{\alpha}^{p}$ as a parameter, and then evolve $\gamma_{\alpha}^{p}$ by Eq. (26). The Maxwell model has the same time 
scale, $t *$, as the Kelvin/Voigt model given by Eq. (23). However, the behaviors are quite different. At $t=0$, there is no viscous shear strain (i.e. $\gamma_{\alpha}^{p}=0$ ), and the model reduces to that for a thin film bonded to an elastic substrate [11]. Thus, for a semi-infinite crack, the stress intensity factor instantaneously takes the form of Eq. (24). When $t \ll t *$, the viscous shear strain $\gamma_{\alpha}^{p}$ is small compared to the elastic strain, and the stress intensity factor is predominantly determined by the elastic solution. When $t \gg t *$, however, the viscous shear strain dominates and the model reduces to the linear viscous model, i.e. Eq. (5). For a semi-infinite crack, in this regime, the stress intensity factor follows Eq. (12), increasing with time indefinitely. Fig. 7(b) shows the schematic of the stress intensity factor as a function of time for a semi-infinite crack if the interlayer is Maxwell-type viscoelastic.

Comparing the viscoelastic behaviors predicted by the Kelvin/Voigt model and the Maxwell Model, we see that fracture in the thin film is constrained according to Kelvin/Voigt model, but the constraint is lost according to the Maxwell model. A more realistic model of viscoelasticity would be the Burger model, which consists of a Maxwell model and a Kelvin/Voigt model in series. We will not discuss the behavior of the Burger model in the present study.

\subsection{Very thick viscous layer}

In addition to the material property of the interlayer, another assumption we made in the present model is that the thickness of the interlayer is small compared to lateral dimensions (e.g. the film size and the crack length). This assumption allows us to further assume a linear variation of the flow velocities across the thickness of the interlayer. When the interlayer is very thick, however, this assumption is not valid and Eq. (5) should be replaced by

$$
\tau_{\alpha}=\eta \frac{\partial \gamma_{\alpha}}{\partial t}
$$

where $\gamma_{\alpha}$ is the viscous shear strain at the interface. To find the shear strain, one has to solve a 3D viscous flow problem. With a semi-infinite viscous layer underneath a thin film, dimensional analysis leads to a time-dependent length scale

$l=E h t / \eta$.

Scaling with this length scale, the stress intensity factor at the tip of a semi-infinite crack takes the form

$K_{\mathrm{I}}(t)=\kappa E \varepsilon_{0}(E h t / \eta)^{1 / 2}$.

Comparing Eq. (29) with Eq. (12), we note the different dependences of the stress intensity factor on the time for a semi-infinite viscous layer and for a thin viscous layer. Thicker viscous layer leads to faster increase in the stress intensity factor. Similarly, for a corner crack in an L-shaped film, Eq. (17) becomes

$K_{\mathrm{I}}(t)=\zeta E \varepsilon_{0}(a)^{1 / 2}\left(\frac{E h t}{\eta a}\right)^{\lambda}$.

The same idea can be extended to the nonlinear creeping model and the viscoelastic models.

\subsection{Ratcheting-creep analogy}

A further extension of the present model considers fracture in thin film structures subjected to ratcheting deformation. Temperature cycling has been used as a reliability test to qualify integrated materials structures in microelectronic and photonic industries. Under certain circumstances, the structures deform in the same direction as the temperature cycles. Such deformation is called ratcheting [18-20]. Several examples of ratcheting in thin film structures have been discovered recently [2126]. In particular, Huang et al. [24] discovered a new failure mechanism induced by ratcheting in thin film structures comprising both ductile and brittle materials and developed a simple model using a ratcheting-creep analogy.

As shown in Fig. 8, thermal expansion mismatch induces a shear stress $\tau_{\mathrm{m}}$ at the top surface of a metal film, which is bonded to an elastic substrate. As temperature cycles, the mismatch of the thermal expansion coefficients between the metal film and the substrate induces biaxial in-plane stresses, which causes the metal film to yield, but the direction of the shear stress does not change. The metal 


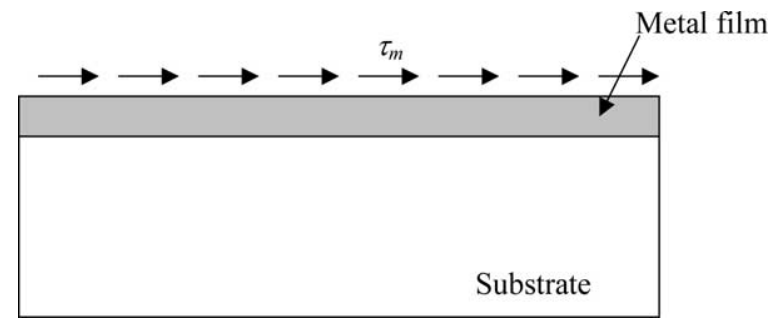

Fig. 8. A metal film subjected to a shear stress at the top surface.

film plastically yields in every temperature cycle, leading to an increment of the plastic shear strain $\gamma^{p}$ in the direction of the shear stress. When the shear stress is small, the ratcheting rate (i.e. the plastic shear strain increment per temperature cycle) is linearly proportional to the shear stress, namely

$\frac{\partial \gamma^{p}}{\partial N}=\frac{\tau_{\mathrm{m}}}{\eta_{\mathrm{R}}}$

where

$$
\eta_{\mathrm{R}}=\frac{E_{\mathrm{m}}}{12\left(1-v_{\mathrm{m}}\right)}\left[\frac{E_{\mathrm{m}}\left(\alpha_{\mathrm{m}}-\alpha_{\mathrm{s}}\right)\left(T_{\mathrm{H}}-T_{\mathrm{L}}\right)}{\left(1-v_{\mathrm{m}}\right) Y}-2\right]^{-1} \text {. }
$$

The proportionality $\eta_{\mathrm{R}}$ is called the ratcheting-viscosity. $E_{\mathrm{m}}$ is the Young's modulus of the metal film, $v_{\mathrm{m}}$ is Poisson's ratio, $Y$ is the uniaxial yield strength, $\alpha_{\mathrm{m}}$ and $\alpha_{\mathrm{s}}$ are the coefficients of thermal expansion of the metal film and the substrate, respectively. The temperature cycles between $T_{\mathrm{L}}$ and $T_{\mathrm{H}}$.

Comparing Eq. (31) with Eq. (5), we see the analogy between ratcheting and creep with the number of cycles $(N)$ in ratcheting analogous to the time $(t)$ in creep and the ratcheting-viscosity $\left(\eta_{\mathrm{R}}\right)$ analogous to the creep viscosity $(\eta)$. Thus, if the interlayer material in Fig. 1(c) is ductile, temperature cycling will induce ratcheting deformation in the ductile layer, which will also cause loss of constraint on fracture in the elastic thin film. The stress intensity factor as a function of the number of temperature cycles follows the similar scaling laws. For a semi-infinite crack, the stress intensity factor takes the form
$K_{\mathrm{I}}(N)=\kappa E \varepsilon_{0}\left(\frac{E h H N}{\left(1-v^{2}\right) \eta_{\mathrm{R}}}\right)^{1 / 4}$,

and for a corner crack in an L-shaped film, we have

$K_{\mathrm{I}}(N)=\zeta E \varepsilon_{0}(a)^{1 / 2}\left[\frac{E h H N}{\left(1-v^{2}\right) \eta_{\mathrm{R}} a^{2}}\right]^{\lambda / 2}$.

\section{Conclusions}

Fracture in a thin elastic film bonded to an elastic substrate is constrained. The constraint is lost if the substrate creeps or if there is a viscous interlayer between the film and the substrate. A 2D model is presented, from which both analytical and numerical solutions are obtained. Extensions of the present model are discussed for nonlinear creeping and viscoelastic interlayers as well as a semi-infinite viscous layer. The analogy between creep and ratcheting for thin film structures under cyclic temperatures is discussed. The present study focuses on stationary cracks. A detailed analysis of the growing cracks will be presented elsewhere [27].

\section{Acknowledgements}

The work is supported by the National Science Foundation through grants CMS-9820713 and CMS-9988788 with Drs Ken Chong and Jorn Larsen-Basse as the program directors, and by the New Jersey Science and Technology Commission. We thank Dr N. Sukumar for helpful discussions about the extended finite element method.

\section{References}

[1] Hutchinson JW, Suo Z. Mixed mode cracking in layered materials. Adv Appl Mech 1992;29:63-191.

[2] Beuth JL. Cracking of thin bonded films in residual tension. Int J Solids Struct 1992;29:1657-75.

[3] Cook RF, Suo Z. Mechanisms active during fracture under constraint. MRS Bull 2002;27:45-51.

[4] Hu MS, Evans AG. The cracking and decohesion of thin films on ductile substrates. Acta mater 1989;37:917-25.

[5] Beuth JL, Klingbeil NW. Cracking of thin films bonded to elastic-plastic substrates. J Mech Phys Solids 1996;44:1411-28. 
[6] Toivola Y, Thurn J, Cook RF. Structural, electrical, and mechanical properties development during curing of low$\mathrm{k}$ hydrogen silsesquioxane films. J Electrochem Soc 2002;149:F9-F17.

[7] Yin H, Huang R, Hobart KD, Suo Z, Kuan TS, Inoki CK et al. Strain relaxation of $\mathrm{SiGe}$ islands on compliant oxide. J Appl Phys 2002;91:9716-22.

[8] Huang R, Yin H, Liang J, Hobart KD, Sturm JC, Suo Z. Relaxation of a strained elastic film on a viscous layer. MRS Symp Proc 2001;695:115-20.

[9] Freund LB, Nix WD. Unpublished work.

[10] Moran PD, Kuech TF. Kinetics of strain relaxation in semiconductor films grown on borosilicate glass-bonded substrates. J Electron Mater 2001;30:802-6.

[11] Xia ZC, Hutchinson JW. Crack patterns in thin films. J Mech Phys Solids 2000;48:1107-31.

[12] Prévost JH. DYNAFLow: a nonlinear transient finite element analysis program, Princeton University; 1981. [Last updated in 2002].

[13] Belytschko T, Black T. Elastic crack growth in finite elements with minimal remeshing. Int J Numer Methods Engng 1999;45:601-20.

[14] Moës N, Dolbow J, Belytschko T. A finite element method for crack growth without remeshing. Int J Numer Methods Engng 1999;46:131-50.

[15] Daux C, Moës N, Dolbow J, Sukumar N, Belytschko T. Arbitrary cracks and holes with the extended finite element method. Int $\mathrm{J}$ Numer Methods Engng 2000;48:1741-60.

[16] Sukumar N, Srolovitz DJ, Baker TJ, Prevost JH. Brittle fracture in polycrystalline microstructures with the extended finite element method. Int J Numer Methods Engng, in press.

[17] Williams ML. Stress singularities resulting from various boundary conditions in angular corners of plates in extension. J Appl Mech 1952;19:526.

[18] Bree J. Elastic-plastic behavior of thin tubes subjected to internal pressure and intermittent high-heat flux with application to fast-nuclear-reactor fuel elements. J Strain Anal 1967;2:226-38.

[19] Jansson S, Leckie FA. Mechanical behavior of a continuous fiber-reinforced aluminum matrix composite subjected to transverse and thermal loading. J Mech Phys Solids 1992;40:593-612.

[20] Suresh S. Fatigue of materials., 2nd ed. Cambridge: Cambridge University Press, 1998.

[21] Begley MR, Evans AG. Progressive cracking of a multilayer system upon thermal cycling. J Appl Mech 2001;68:513-20.

[22] He MY, Evans AG, Hutchinson JW. The ratcheting of compressed thermally grown thin films on ductile substrates. Acta mater 2000;48:2593-601.

[23] Huang M, Suo Z, Ma Q, Fujimoto H. Thin film cracking and ratcheting caused by temperature cycling. J Mater Res 2000;15:1239.

[24] Huang M, Suo Z, Ma Q. Metal film crawling in interconnect structures caused by cyclic temperatures. Acta mater 2001;49:3039-49.

[25] Huang M, Suo Z, Ma Q. Plastic ratcheting induced cracks in thin film structures. J Mech Phys Solids 2002;50:1079-98.

[26] Karlsson AM, Evans AG. A numerical model for the cyclic instability of thermally grown oxides in thermal barrier systems. Acta mater 2001;49:1793-804.

[27] Liang J, Huang R, Prevost JH, Suo Z. Concomitant thin film cracking and underlayer creep, submitted for publication. Preprint available online at http:// www.princeton.edu/ suo, Publication 132, 